\title{
Excitation of the third harmonic mode in meridian planes for $\mathrm{Pi} 2$ in the auroral zone
}

\author{
O. Saka, ${ }^{1}$ K. Hayashi, ${ }^{2,3}$ and D. Koga ${ }^{4}$ \\ Received 6 June 2012; revised 23 September 2012; accepted 16 October 2012; published 12 December 2012.
}

[1] Polarizations of Pi2 pulsations in the magnetosphere and on the ground in the auroral zone are inconsistent when field line motions in the meridian and transverse planes are both in the fundamental harmonic. To resolve these inconsistencies, we propose a third harmonic mode in the meridian planes. The excitation of the third harmonic is explained by dusk-to-dawn currents at the equatorial plane, which are driven by diamagnetic currents during substorm injections. We propose diamagnetic currents in the equatorial plane and compressional input at the outer boundary as the source of $\mathrm{Pi} 2$ pulsations in the magnetosphere.

Citation: Saka, O., K. Hayashi, and D. Koga (2012), Excitation of the third harmonic mode in meridian planes for Pi2 in the auroral zone, J. Geophys. Res., 117, A12215, doi:10.1029/2012JA018003.

\section{Introduction}

[2] Nighttime Pi2 pulsations are geomagnetic oscillations in space observed in the plasmasphere [Takahashi et al., 1992, 2003] and outside the plasmapause [Kim et al., 2005; Teramoto et al., 2008]. Pi2 pulsations are also observed on the ground at a wide range of latitudes from the dip equator to the polar cap [Shinohara et al., 1997; Li et al., 1998; Samson and Harrold, 1983; Uozumi et al., 2000]. Following the publication of Saito [1961], Pi2 pulsations were a subject of intense interest for many decades due to their close association with the substorm expansion onset and their global distributions on the ground and in space. Pi2 models that have been proposed include (1) plasmaspheric cavity resonance [Allan et al., 1996; Li et al., 1998], (2) plasmaspheric virtual resonance [Lee, 1998; Lee and Lysak, 1999] and (3) ionospheric current oscillations linked to the field-aligned currents [Samson, 1982; Lester et al., 1983; Baumjohann and Glassmeier, 1984]. A comprehensive review of Pi2 models can be found in Keiling and Takahashi [2011].

[3] To explain the signal source of Pi2s, an external stimulus propagating in azimuth at the outer boundary of the inner magnetosphere with low $m$ number was proposed. Using the compressional input for the external stimulus, Lee and Lysak [1991] and Allan et al. [1996] simulated Pi2 propagations across the plasmasphere and studied the spatiotemporal distributions of ground Pi2 signals. From ground observations, $\mathrm{Pi} 2 \mathrm{~s}$ propagate either westward or eastward at angular velocities on the order of $0.5-2$ degrees/s corresponding to $m=2-7$,

\footnotetext{
${ }^{1}$ Office Geophysik, Ogoori, Japan.

${ }^{2}$ Earth and Planetary Physics, University of Tokyo, Tokyo, Japan.

${ }^{3}$ Retired.

${ }^{4}$ National Institute for Space Research, Sao Jose dos Campos, Brazil.

Corresponding author: O. Saka, Office Geophysik, Ogoori 838-0141, Japan. (saka.o@nifty.com)

(C2012. American Geophysical Union. All Rights Reserved. 0148-0227/12/2012JA018003
}

without significant changes from high to low latitudes [Uozumi et al., 2004; Samson and Harrold, 1985; Lester et al., 1984; Li et al., 1998]. The external stimulus proposed by Allan et al. [1985] and Lee and Lysak [1991] are suggested to correspond to the bidirectional flow burst at the outer boundary of the inner magnetosphere. The earthward flow burst and associated bidirectional flow could be a process associated with the tail current disruption [Saka et al., 2012].

[4] In this report, we study waveform comparisons of Pi2s using magnetometer data from a geosynchronous satellite as well as from ground stations located at the satellite footprint in the auroral zone. We determined the harmonic of standing modes in the transverse and meridian planes in the magnetosphere and interpreted the Pi2s in terms of the Cavity mode. The present model also explained the ground Pi2 signals in the auroral zone within the framework of ionospheric amplification of Cavity mode without employing the field-aligned currents and associated ionospheric conductivity enhancement.

\section{Observations}

[5] In this report, we study the ground-satellite conjunctions of magnetometer data using a geosynchronous satellite (GOES 5, L =6.6) as well as ground stations in the auroral zone (GWR, $\mathrm{L}=6.3$ ) and in the dip equator (HUA, $\mathrm{L}=1.05$ ). In the IGRF (International Geomagnetic Reference Field) model, the geographic coordinates of the satellite footprints, GWR, and HUA are (56.3 N, 281.4), (55.3 N, 282.2), and (12.1 S, 284.7), respectively. The footprints in the T89 model [Tsyganenko, 1989] are $(54.9 \mathrm{~N}, 281.4)$ for $\mathrm{Kp}=0$ in geographic coordinates. We examined three events during the Global Aurora Dynamics Campaign [Oguti et al., 1988]. The onsets of these events were 0444 UT (23.6 MLT at GWR) for the 24 January event, 0501 UT (23.9 MLT) for the 27 January event, and 0517 UT $(0.2 \mathrm{M} \mathrm{LT})$ for the 30 January event. During each event, an auroral surge was observed at the conjugate meridian propagating along the poleward boundary of the auroral zone. The surge propagated periodically at 


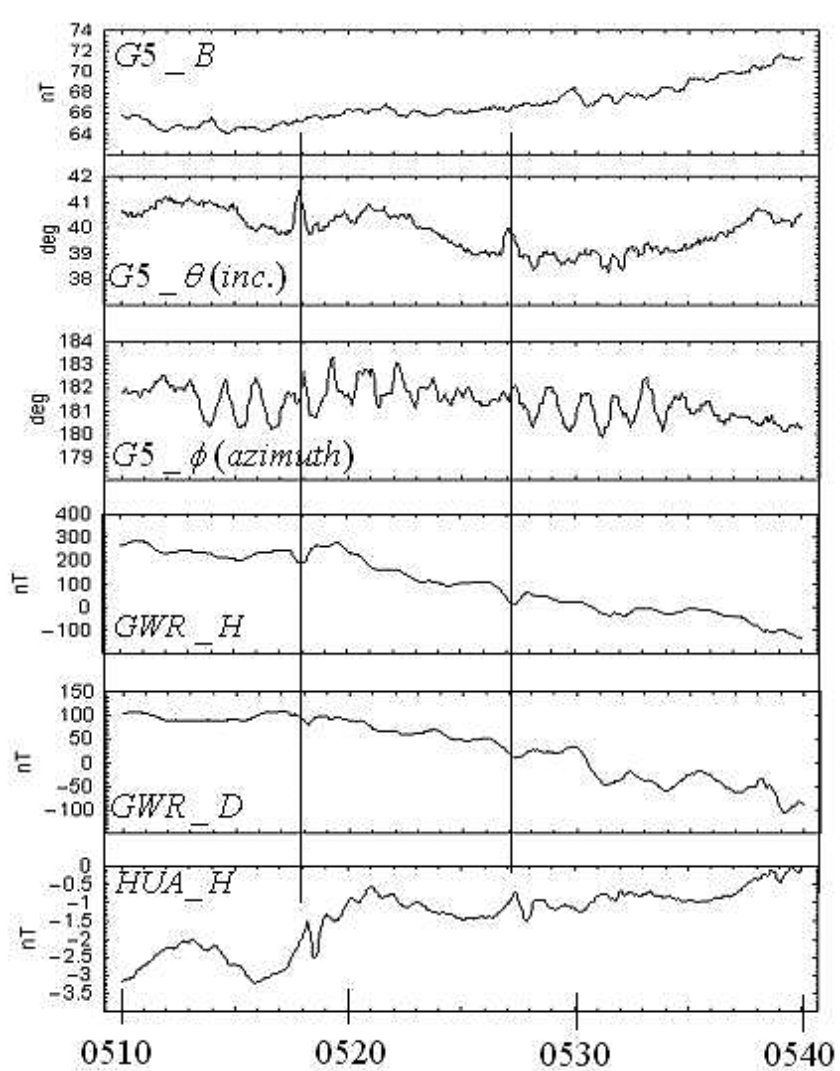

Figure 1. Combined $30 \mathrm{~min}$ plots for the 30 January event (0510-0540 UT). From top to bottom: field magnitudes (B) in $\mathrm{nT}$, inclination angle $(\theta)$, and azimuth angle $(\phi)$ in degrees for GOES 5 at geosynchronous altitudes, $\mathrm{H}$ at GWR in the auroral zone, D at GWR, and $\mathrm{H}$ at HUA at the dip equator. The vertical bars indicate two consecutive onsets of single dipolarization observed by GOES 5. Background levels were removed from the ground magnetometer data.

the Pi2 periodicities eastward for the 24 January event and westward for the 27 January event. For the 30 January event, a single passage of the auroral surge propagating westward was observed. The auroral surge is suggested to be an auroral manifestation of the bidirectional flow burst at the outer boundary [Saka et al., 2012].

[6] Satellite magnetometer data were studied in the polar coordinates $(\theta, \phi)$, where $\theta$ is the inclination angle measured northward from the V-D plane and $\phi$ represents the azimuth in the V-D plane measured counterclockwise from the V axis. $\mathrm{H}$ is measured northward parallel to the dipole axis, $\mathrm{V}$ is radial outward, and D is dipole east. In the polar coordinates, the azimuth angle is 180 when the field line vector points toward Earth. The field line motions associated with the circular rotations (not the twist) of the plasma slabs at the equatorial plane may cause wave polarizations, $\delta B_{\perp}$, in the satellite magnetometer data off the equatorial plane [Saka et al., 2007]. $\delta B_{\perp}$ is written as $\left(B_{0} \delta \theta, B_{0} \delta \phi\right)$, where $B_{0}$ represents the mean magnitudes. We used $(\delta \theta, \delta \phi)$ instead of $\left(B_{0} \delta \theta, B_{0} \delta \phi\right)$ for the polarization analyses. Angle changes on the order of 2 degrees in polar coordinates correspond to
$3.0 \mathrm{nT}$ for $\delta B_{\perp}$ at geosynchronous altitudes for mean field magnitudes of $85.3 \mathrm{nT}$ at the Pi2 onset [Saka et al., 2010]. For the ground magnetometer data, $(H, D, Z)$ coordinates were used, where $H$ is local magnetic north, $Z$ is positive downward, and $D$ is perpendicular to the $\mathrm{H}-\mathrm{Z}$ plane and positive eastward.

\subsection{The 30 January 1986 Event}

[7] Figure 1 shows a $30 \mathrm{~min}$ long plot of satellite data (GOES 5) and ground magnetometer data at the auroral zone (GWR) and at the dip equator (HUA). GOES 5 observed two single pulse dipolarization events at $0518 \mathrm{UT}$ and at 0527 UT in the midnight sector. In the azimuth component of the satellite data, background fluctuations can be observed in the

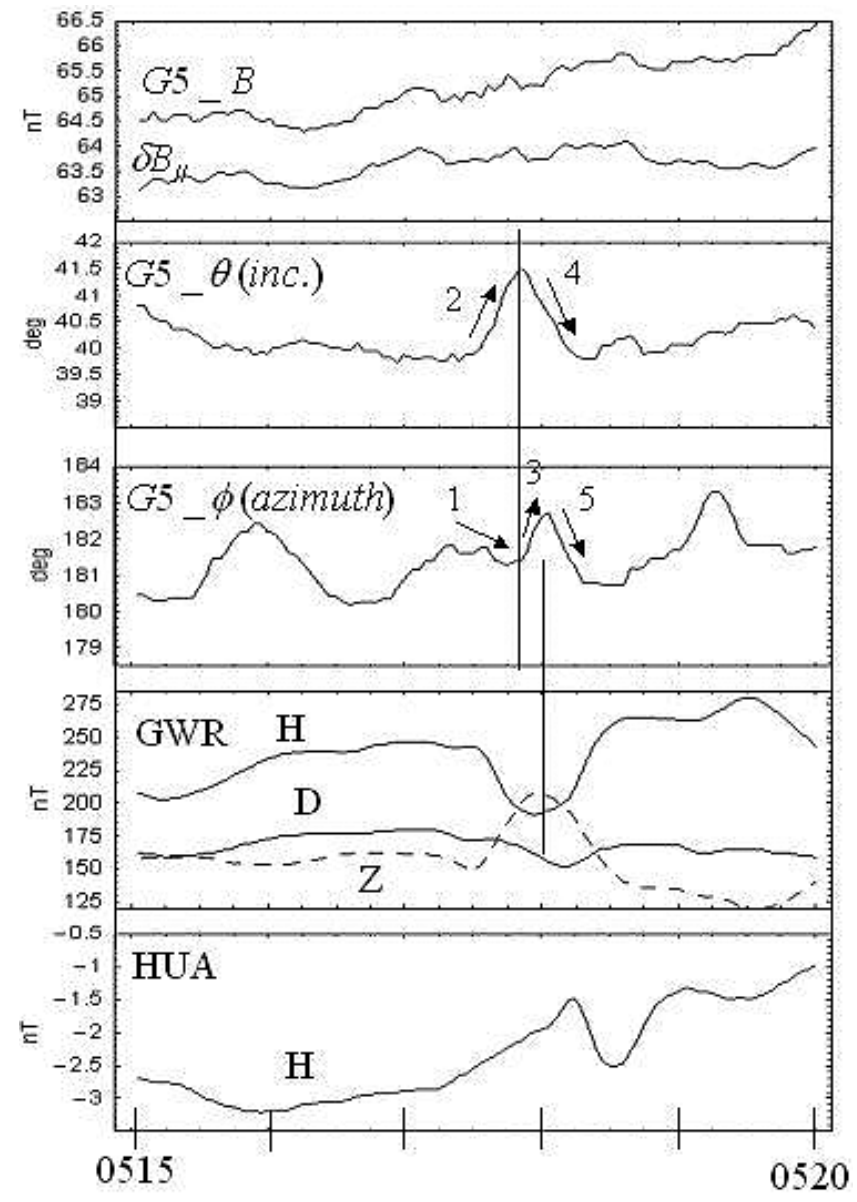

Figure 2. Combined $5 \mathrm{~min}$ plots for the 30 January event (0515-0520 UT) in the same format as Figure 1, except that (1) GOES 5 shows field magnitude $(B)$ and $\delta B_{\|}$(band-pass filtered from 1 to $100 \mathrm{mHz}$ ) and (2) GWR shows three components: $\mathrm{H}, \mathrm{D}$, and $\mathrm{Z}$. The field magnitudes $B$ and $\delta B_{\|}$are shown at the same scale but an offset was added to the $\delta B_{\|}$. The vertical component $(\mathrm{Z})$ is shown by the dotted line. The first vertical line indicates the peak of dipolarization observed by GOES 5 (0517:49 UT). The second line indicates the peak of westward deflection in the azimuth angle observed by GOES 5 (0518:00 UT). Background levels were removed from the ground magnetometer data. 


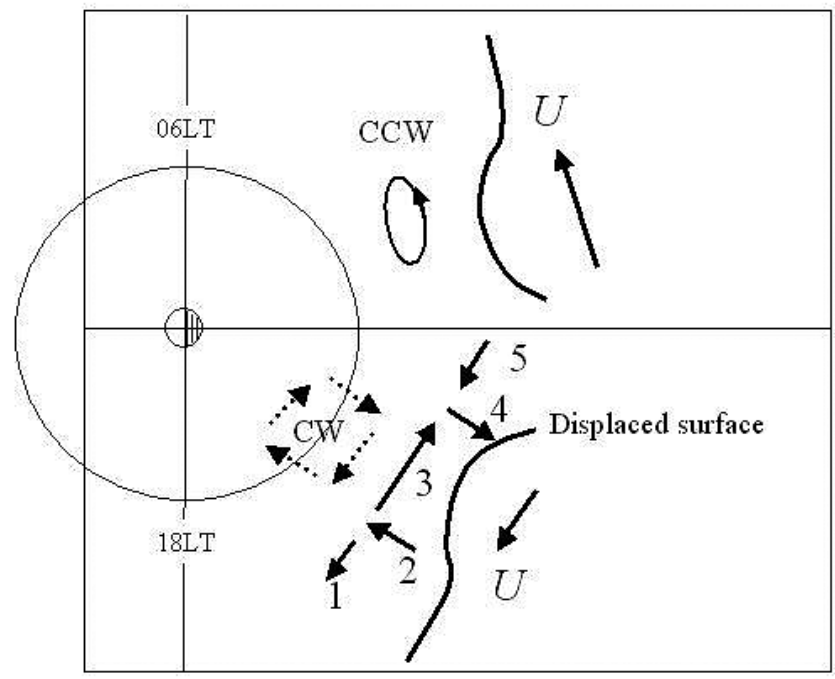

Figure 3. Schematic illustration of surface displacement in the equatorial plane at the passage of bidirectional flow at the outer boundary. The bidirectional flow passed at the velocity $U$. The consecutive plasma displacement associated with the passage is marked 1 through 5. Refer to Saka et al. [2012] for details.

Pi2 band starting at 0513 UT. The dipolarization pulse interrupted the background fluctuations. Pi2 pulsations can be observed at the dip equator related to the dipolarization at 0518 UT and 0527 UT. In the auroral zone, a negative pulse can be observed in the $\mathrm{H}$ and $\mathrm{D}$ components. We closely

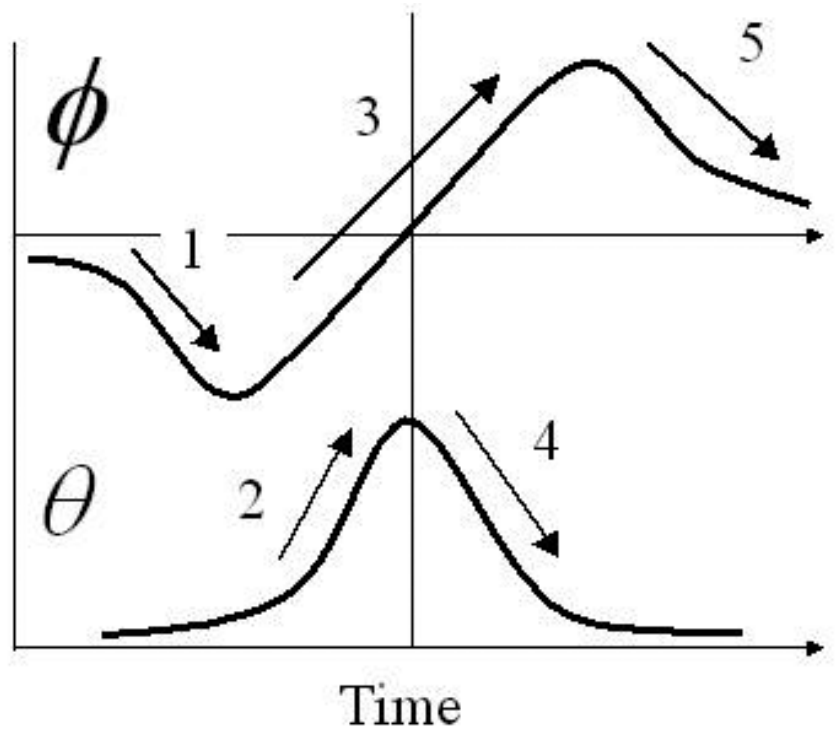

Figure 4. Plot of the field variations in the azimuth $(\phi)$ and inclination $(\theta)$ angles north of the equatorial plane during the westward propagation of the flow burst. Numbers 1 through 5 correspond to the field changes associated with the plasma displacement 1 through 5 in Figure 3.

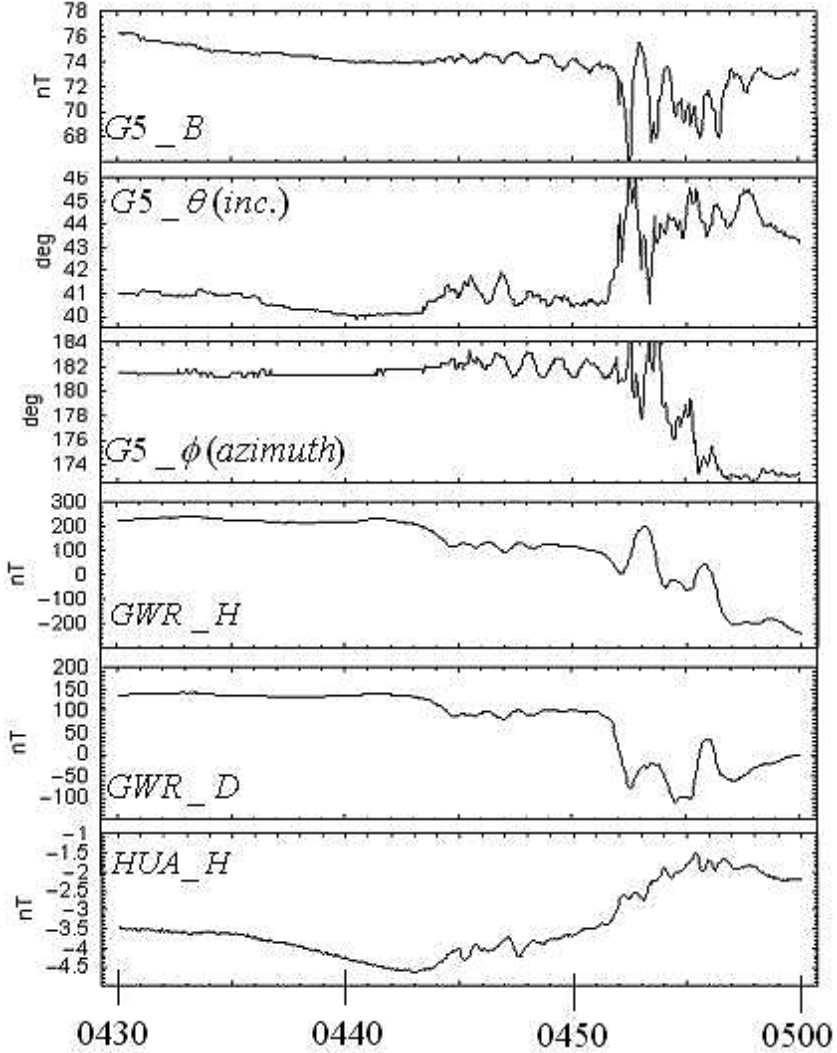

Figure 5. Same as Figure 1 but for the 24 January event (0430-0500 UT).

examined the first dipolarization event using an enlarged time scale.

[8] Figure 2 combines $5 \mathrm{~min}$ long plots from $0515 \mathrm{UT}$ to 0520 UT for the first dipolarization event. The dipolarization pulse of GOES 5 (0517:50 UT) preceded the initial peak of Pi2 at the dip equator by $20 \mathrm{~s}(0518: 10 \mathrm{UT})$. We conclude that the $\mathrm{Pi} 2$ pulsations observed at the dip equator were related to the dipolarization onset at the equatorial plane. In association with the dipolarization pulse, a bipolar change can be observed in the azimuth component $(\phi)$. Field variations in the azimuth $(\phi)$ and inclination $(\theta)$ components are labeled 1 through 5 . These vector changes can be interpreted as the westward passage of the bidirectional flow at the outer boundary (see Figures 3 and 4). The first negative pulse of $\phi$, labeled 1 and 3 , corresponds to the passage of the leading edge of the westward flow, and the subsequent positive pulse, labeled 3 and 5, corresponds to the passage of the trailing edge. Bipolar variations in $\phi$ show an amplitude asymmetry with respect to the dipolarization peak in $\theta$. The dipolarization pulse, labeled 2 and 4, represents the inward displacement of the outer boundaries by the passage of the bidirectional flow. Passage of the bidirectional flow at $\mathrm{L}=10$ with a velocity of $300 \mathrm{~km} / \mathrm{s}$ and an azimuth scale on the order of $4.7 \mathrm{Re}(\mathrm{m}=6.7)$ may invoke a $100 \mathrm{~s}$ dipolarization pulse.

[9] At the satellite footprint in the auroral zone (GWR), vector changes in the $H$ and $D$ components were observed with time delays of approximately $7 \mathrm{~s}$ with respect to those 


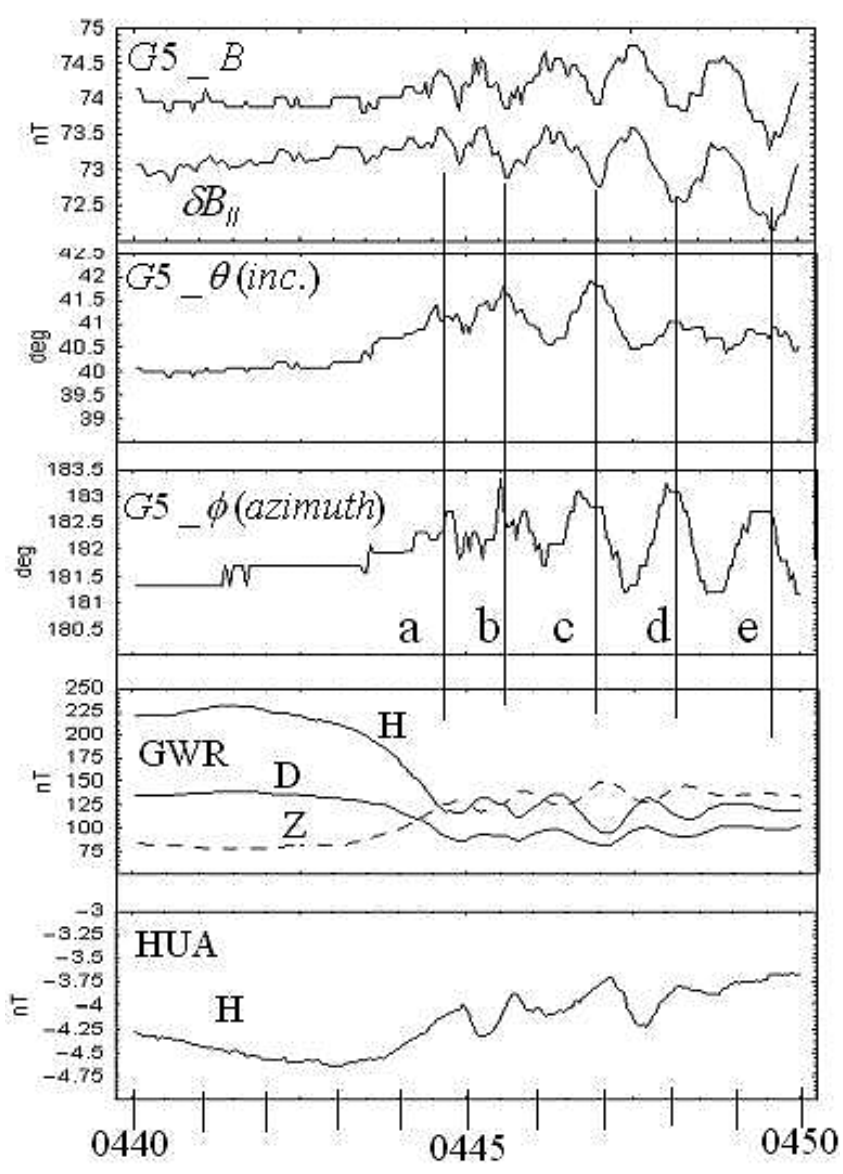

Figure 6. Same as Figure 2 but for the 24 January event (0440-0450 UT). The vertical lines (a, b, c, d, and e) indicate dipolarization peaks observed by GOES 5 .

marked by vertical bars at GOES 5, although the polarities were reversed at GWR. The $\mathrm{Z}$ component oscillated antiparallel to H. Time delays of $20 \mathrm{~s}$ in the dip equator and $7 \mathrm{~s}$ in the auroral zone can be understood by the Alfven transit time from $\mathrm{L}=6.6$ in the dip equator across the equatorial plane at a mean velocity of $1760 \mathrm{~km} / \mathrm{s}$ and in the auroral zone along the field lines at a mean velocity of $7400 \mathrm{~km} / \mathrm{s}$.

[10] The initial increase in $\mathrm{H}$ at the dip equator (HUA) started at 0516:00 UT, which was 2 min prior to the dipolarization pulse at GOES 5. Thereafter, the rate of amplitude change increased at 0517:14 UT. The second onset developed to the Pi2 pulse, although the first onset did not develop to the $\mathrm{Pi} 2$ pulse.

\subsection{The 24 January 1986 Event}

[11] Figure 5 shows a 30 min plot of satellite data (GOES 5) and ground magnetometer data at the auroral zone (GWR) and at the dip equator (HUA). GOES 5 observed $\mathrm{Pi} 2$ events in the midnight sector from 0443 UT to 0451 UT and subsequently from $0452 \mathrm{UT}$ to $0458 \mathrm{UT}$. For both of these intervals, an auroral surge was observed at the poleward boundary. We examine $\mathrm{Pi} 2 \mathrm{~s}$ in the first interval where the surge repeated and correlated to the Pi2 pulse at GOES 5 and on the ground (refer to Saka et al. [2012] for details). Ten min long plots (0440-
0450 UT) are presented in Figure 6 using the same format as Figure 2. The initial increase in $\mathrm{H}$ at the dip equator started at 0443:00 UT (Figure 6), and it oscillated for about four cycles at periods of 50-80 s. Periodic dipolarization was simultaneously recorded by the GOES 5 magnetometer with a peak preceding the Pi2 peak by $15 \mathrm{~s}$ at the dip equator. At the auroral zone, the amplitudes of $\mathrm{H}$ began to decrease at 0441:30 UT simultaneous with the $\mathrm{Z}$ increase. $\mathrm{H}$ and $\mathrm{Z}$ oscillated out of phase correlating to the dipolarization at the geosynchronous altitudes with time delays of approximately $10 \mathrm{~s}$. D in the auroral zone (GWR) and $\phi$ at geosynchronous altitudes oscillated out of phase with time delays of approximately $10 \mathrm{~s}$. In other words, the vector changes in H and D were observed at the satellite footprint in the auroral zone (GWR) with time delays of approximately $10 \mathrm{~s}$ with respect to those at GOES 5, although the polarities were reversed at GWR. Pre-onset signs at the dip equator (HUA) can be observed at 0443:00 UT and at 0443:47 UT. The second onset is associated with the onset of dipolarization at GOES 5. The 24 January event may be attributed to consecutive passages of eastward flows (invert the bipolar change in the azimuth angle in Figure 4) because, except for the vertical bars "a" and "b," the inclination pulses delayed the azimuth pulse. We suggest that the pulses in the inclination and azimuth angles at the geosynchronous altitudes were transmitted by Alfven waves to the auroral zone (GWR) with $10 \mathrm{~s}$ delays and to the dip equator with 15 s delays.

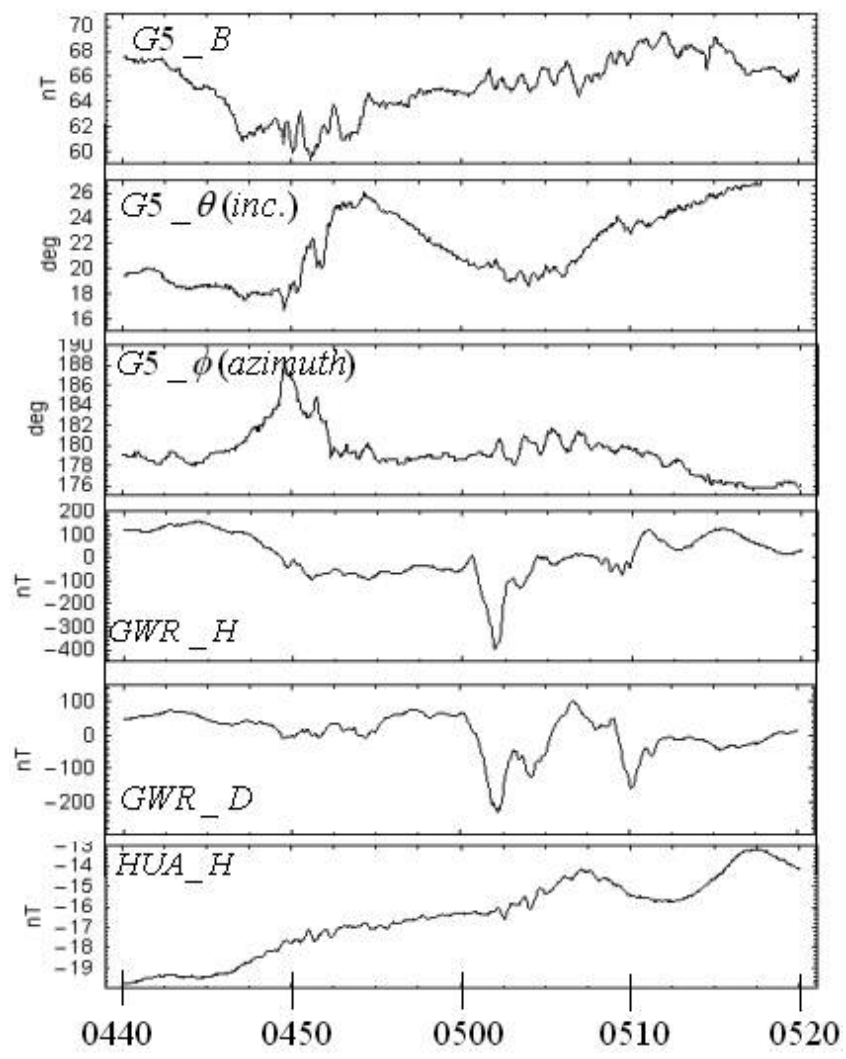

Figure 7. Same as Figure 1 but for the 27 January event (0440-0520 UT). 


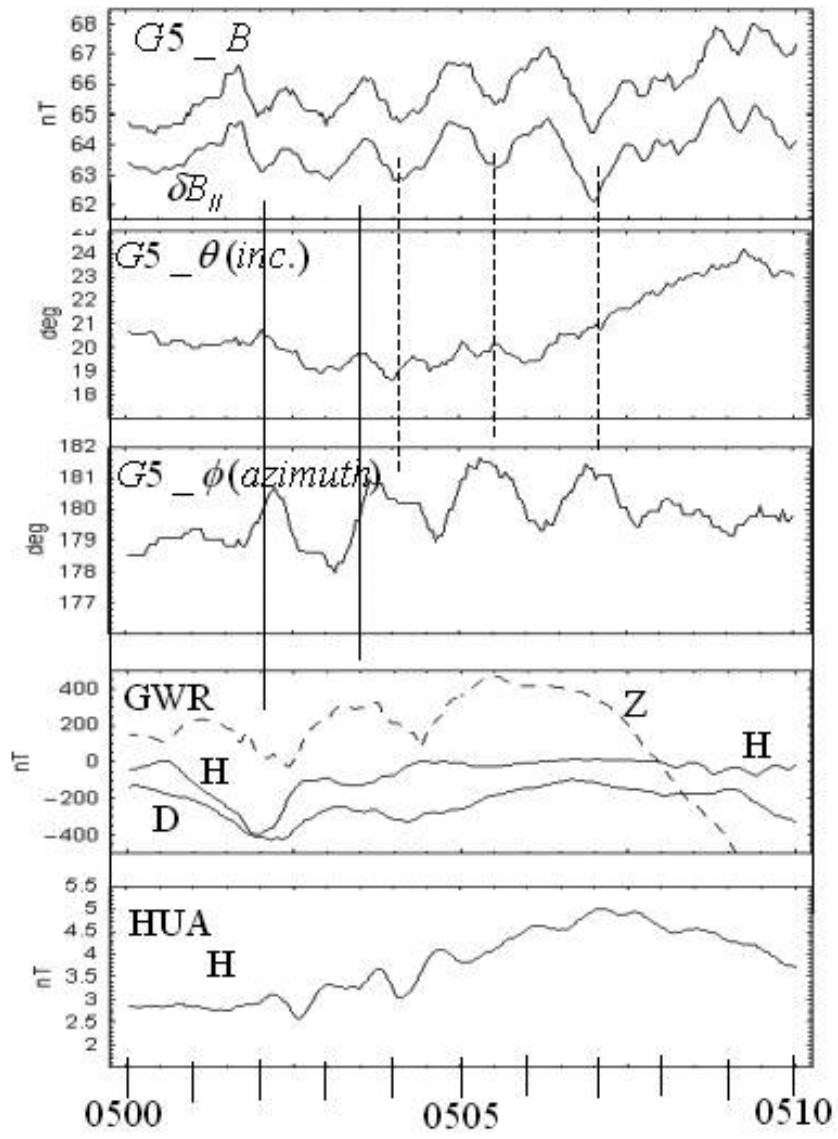

Figure 8. Same as Figure 2 but for the 27 January event (0500-0510 UT). The solid vertical lines indicate the first two dipolarization peaks observed by GOES 5 . The dotted lines show peaks in the azimuth angles correlated to the depression of the field magnitudes.

\subsection{The 27 January 1986 Event}

[12] Figure 7 shows a $40 \mathrm{~min}$ long plot of satellite data (GOES 5) and ground magnetometer data at the auroral zone (GWR) and at the dip equator (HUA). We examined the Pi2 activities from 0500 to $0510 \mathrm{UT}$, when the auroral surge

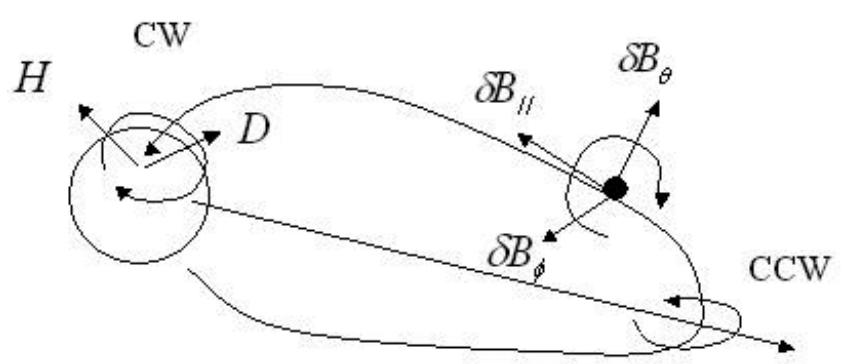

Figure 9. Illustrations of the clockwise rotation of field line vectors for fundamental harmonic in both the toroidal $(\phi)$ and poloidal $(\theta)$ components. The vector components $\left(\delta B_{\|}\right.$, $\left.\delta B_{\theta}, \delta B_{\phi}\right)$ represent field line vectors in polar coordinates. The rotations are mapped in the H-D plane on the ground as the $\mathrm{CW}$ polarizations. At the equator, mapped polarizations are $\mathrm{CCW}$ when viewed from the north.
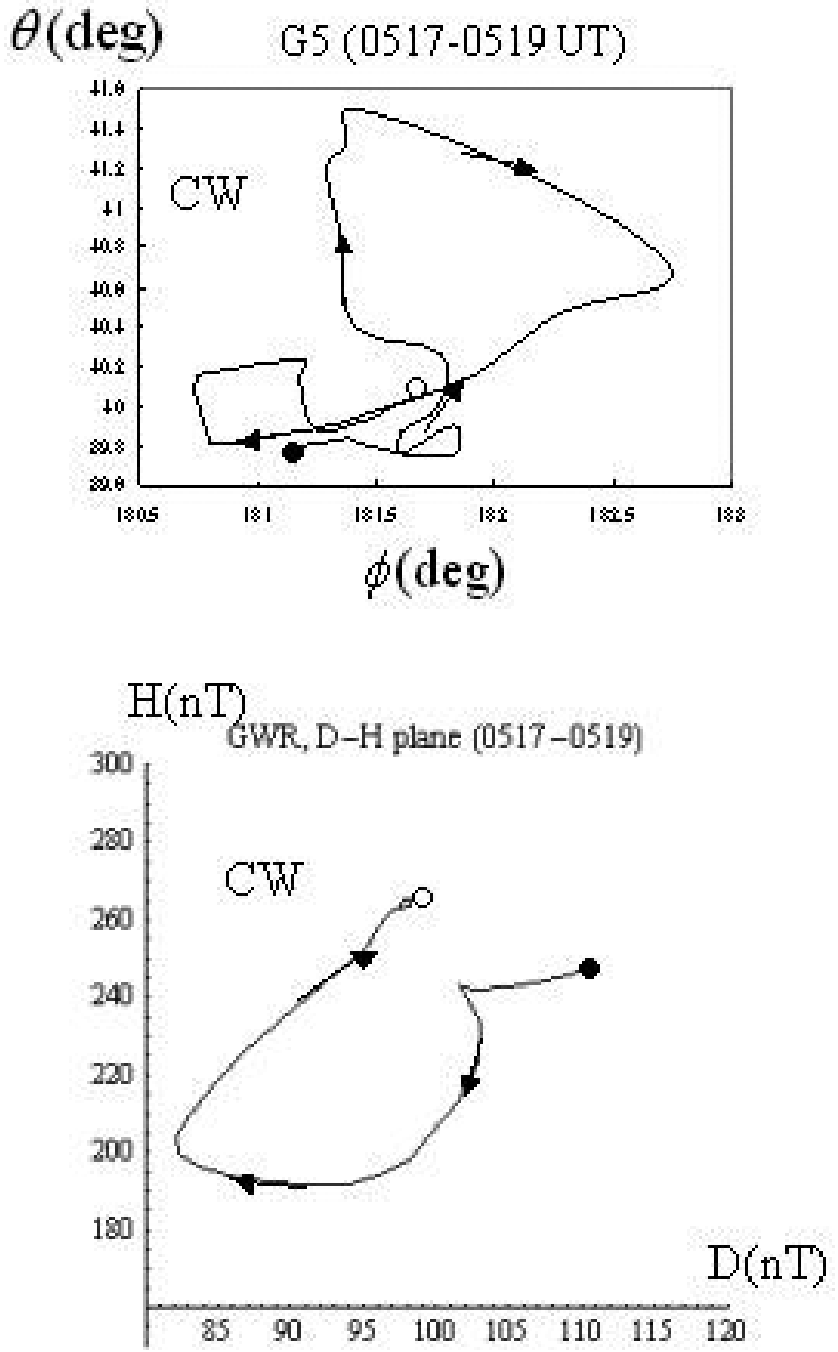

Figure 10. (top) Hodogram of the field line vector in the $\phi-\theta$ plane of GOES 5 for the interval 0517-0519 UT, 30 January. (bottom) Hodogram of the field line vector in the H-D plane of the auroral zone station (GWR) for the same interval, 0517-0519 UT. The plots begin at the black circle and end at the white circle. The arrows indicate consecutive changes in the field line vectors. The sense of polarization is CW for both GOES 5 and GWR.

repeated at the poleward boundary at $\mathrm{Pi} 2$ periodicities. Combined $10 \mathrm{~min}$ plots (0500-0510 UT) are presented in Figure 8 using the same format as Figure 2. Although the waveforms are irregular, the $\mathrm{Pi} 2$ peaks at the dip equator correlate to the dipolarization at the geosynchronous altitudes with time delays of approximately $9 \mathrm{~s}$. The value of $\mathrm{H}$ in the auroral zone decreased in association with the dipolarization at the geosynchronous altitudes, as marked by the vertical bars. The value of $\mathrm{Z}$ tended to increase in association with the decrease in $\mathrm{H}$. There were no apparent time delays for the ground pulses with respect to those recorded by the satellite, except for the second decrease in D (0504:15 UT). The decrease in D was $20 \mathrm{~s}$ after the positive pulse in the azimuth angles at the equatorial plane. The first 


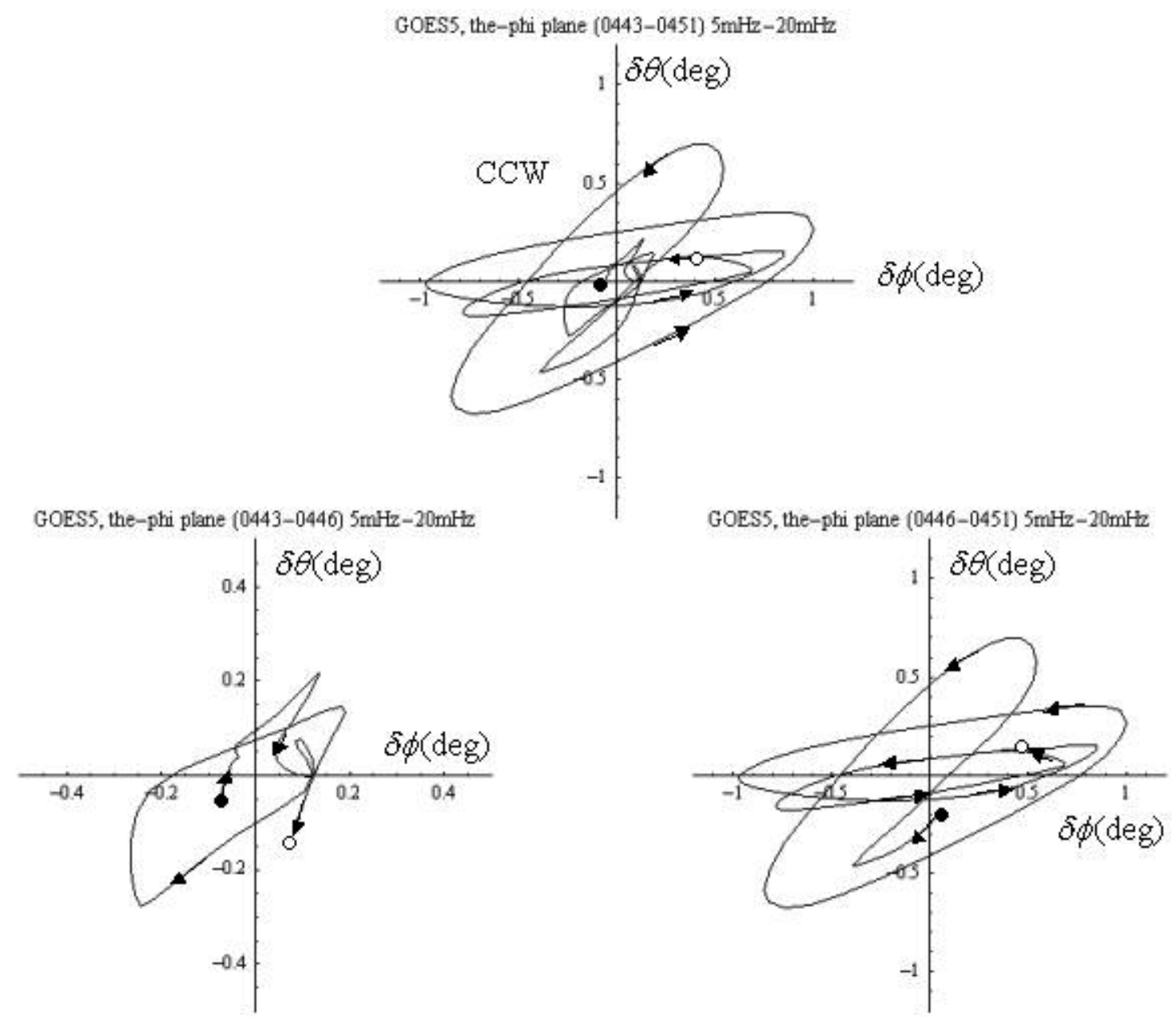

Figure 11. Same as Figure 10 but for the interval 0443-0451 UT, 24 January for GOES 5. Data were band-pass filtered from 5 to $20 \mathrm{mHz}$. In the bottom panel, the interval 0443-0451 UT is divided into (left) 0443-0446 UT and (right) 0446-0451 UT. The sense of polarization was CW for the first 3 min interval and $\mathrm{CCW}$ in the following $5 \mathrm{~min}$ interval. Note the different amplitudes in the bottom panel.

two pulses in the inclination angle, as marked by vertical bars, may be attributable to consecutive passages of westward flows because the inclination leads the azimuth pulse. Although the polarities were reversed, we suggest that pulses at the geosynchronous altitudes in the inclination and azimuth angles for this event were also transmitted by Alfven waves to the ground. They were recorded in the $\mathrm{H}$ and $\mathrm{D}$ components of GWR with 0 to 20 s delays.

\subsection{Polarization Analyses}

[13] Figure 9 shows how the rotation of the field line vectors observed by the satellite off the equatorial plane are mapped to the equatorial plane in the magnetosphere and on the ground, when the fundamental harmonic is assumed for both the toroidal $(\phi)$ and poloidal components $(\theta)$. Ground polarizations are plotted in the H-D plane. Satellite polarizations are illustrated by $\delta B_{\theta}$ and $\delta B_{\phi}$. Projected polarizations are counterclockwise $(\mathrm{CCW})$ when viewed antiparallel to the field lines at the equatorial plane and clockwise $(\mathrm{CW})$ when viewed parallel to the field lines on the ground. Figure 10 (top) shows a hodogram of the magnetic field vectors in the $\theta-\phi$ plane of GOES 5 during the 30 January (0517-0519 UT) dipolarization event. This hodogram suggests that the field line vector rotated clockwise $(\mathrm{CW})$ around the mean fields as viewed in the opposite direction of the field lines. The plasma slab in the equatorial plane is thought to have slipped in the same sense, i.e., clockwise as viewed from the north [Saka et al., 2007]. At the satellite footprint (GWR), CW polarization was observed in the H-D plane (Figure 10, bottom). Figure 11 shows $\mathrm{Pi} 2$ hodograms in the $\theta-\phi$ plane for GOES 5 (band-pass filtered from 5 to $20 \mathrm{mHz}$ ) and Figure 12 shows Pi2 hodograms in the H-D plane for GWR each for the 24 January event. The upper hodogram (0443-0451 UT) is divided into two intervals; 0443-0446 UT (bottom left) and 0446-0451 UT (bottom right). The first two pulses (0443-0446 UT) were polarized $\mathrm{CW}$ and the last three pulses (0446-0451 UT) were polarized $\mathrm{CCW}$ in both the $\theta-\phi$ and H-D planes. The first interval included the onsets of dipolarization marked by "a" and " $b$ " in Figure 6. For the 27 January event shown in Figures 13 and 14, the sense of the polarization was CW in both the $\theta-\phi$ and $\mathrm{H}-\mathrm{D}$ planes, although some irregularity interrupted the $\mathrm{CW}$ 

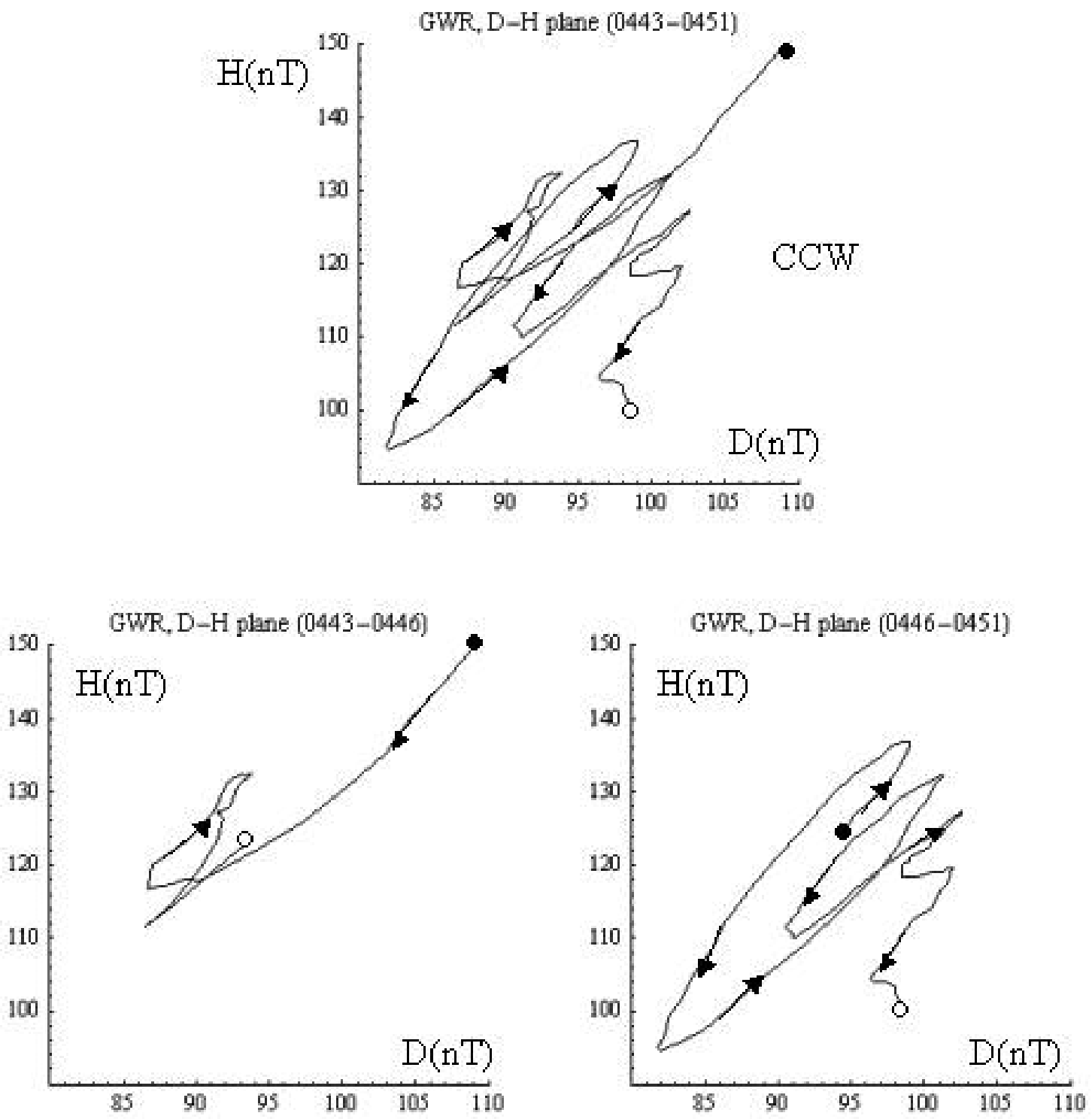

Figure 12. Same as Figure 10 but for GWR, using unfiltered data. In the bottom panel, the interval 0443-0451 UT is divided into (left) 0443-0446 UT and (right) 0446-0451 UT. The sense of polarization was CCW except for the first 3 min interval.

polarization in the $\theta-\phi$ plane. Consequently, the sense of rotation of the field line vectors around the mean fields between the geosynchronous altitudes and on the ground cannot be interpreted if we assume that both the toroidal and poloidal components are in the fundamental harmonic.

\section{Discussion}

[14] The time delays observed between the satellite magnetometer data at geosynchronous altitudes and those on the ground station (7 to $20 \mathrm{~s}$ ) should represent the transit time of the Alfven wave. Because the Alfven velocities are lowest at the equatorial plane, the transit time is longer for the propagation across the equatorial plane to the dip equator than propagation along the field lines to the auroral zone. For the
30 January event, the mean Alfven velocities between $\mathrm{L}=6.6$ and the dip equator were $1760 \mathrm{~km} / \mathrm{s}$, while those along the field lines to the auroral zone were $7400 \mathrm{~km} / \mathrm{s}$, which is consistent with the local Alfven velocities at the equatorial plane [Lee and Lysak, 1999] and along the high-latitude orbit near local midnight [Wygant et al., 2000], respectively.

[15] The Pi2 amplitudes recorded at the ground station in the auroral zone ranged between $20 \mathrm{nT}$ (for the $\mathrm{D}$ component of the 30 January event) to $400 \mathrm{nT}$ (for the $\mathrm{H}$ component of the 27 January event), while the Pi2 amplitudes were $\sim 1 \mathrm{nT}$ at the dip equator. The Pi2 amplitudes exceeding $50 \mathrm{nT}$ were often observed at high-latitude stations [Samson and Harrold, 1983]. The Pi2 signals at the auroral zone may be amplified. We assume that the discontinuities between field 

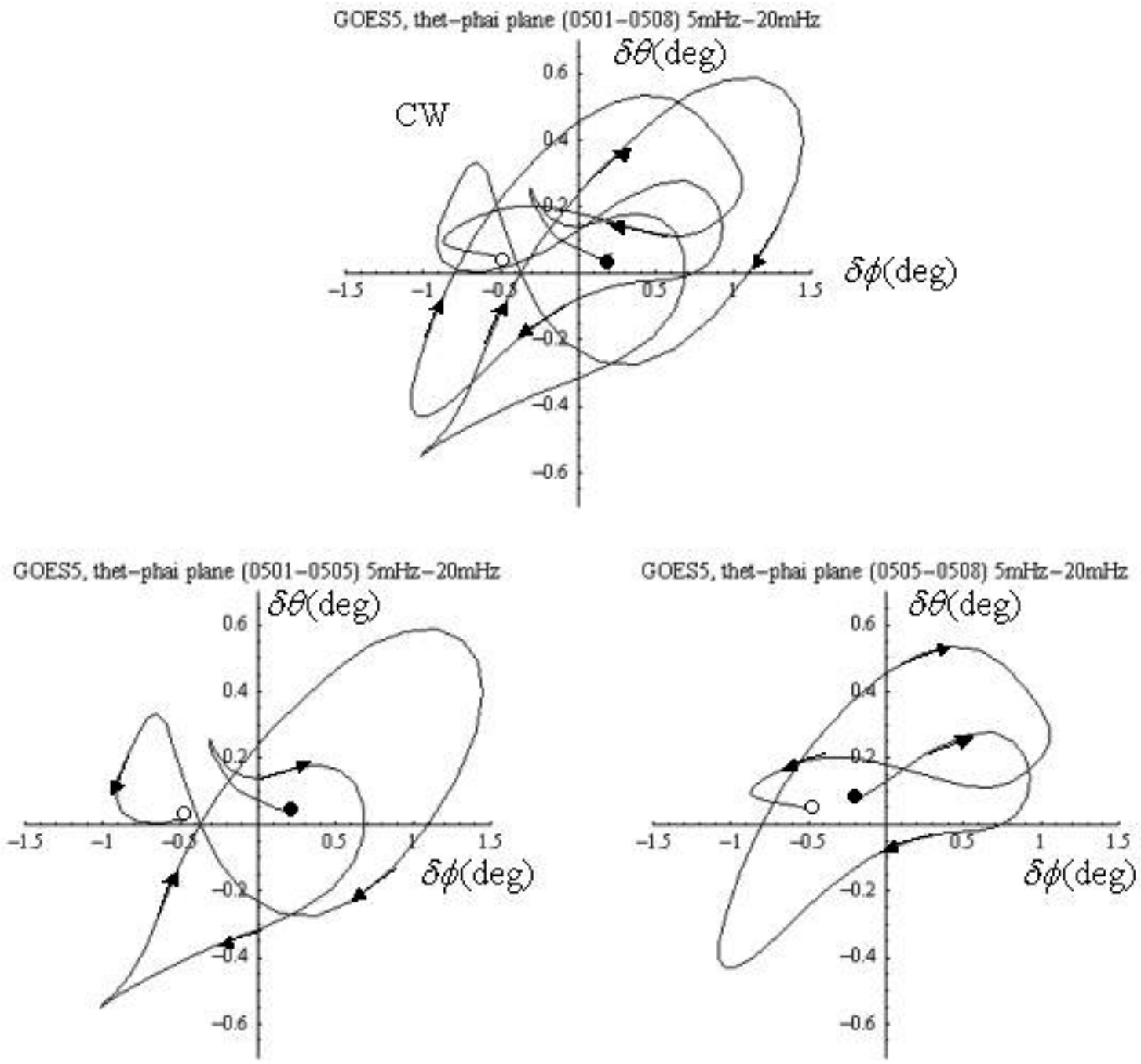

Figure 13. Same as Figure 10 but for the interval 0501-0508 UT, 27 January for GOES 5. Data were band-pass filtered from 5 to $20 \mathrm{mHz}$. In the bottom panel, the interval 0501-0508 UT is divided into (left) 0501-0505 UT and (right) 0505-0508 UT. The sense of polarization was CW. Note that the hodograms are plotted in different scales in the vertical and horizontal axes.

lines in the atmosphere and those at the earthward boundary of the magnetosphere are a result of the field line motions in the magnetosphere. To smooth out the discontinuities, the Hall currents were excited in the ionosphere by the local electric fields generated at the magnetosphere-atmosphere interface. There may be no significant time delays for the development of the Hall currents in the ionosphere because field line discontinuities at the magnetosphere-atmosphere interface must be canceled promptly. The ionospheric Hall currents that cancel the field line discontinuities in transverse planes (toroidal component) and in meridian planes (poloidal component) at the magnetosphere-atmosphere interface may form a current loop in the ionosphere.

[16] The vector change of field lines in terms of inclination and azimuth at GOES 5 propagated to the conjugate ground station. Figure 15 shows how the vector changes at GOES 5 relate to those at the auroral zone. Figure 15 (top) illustrates the westward deflection of the azimuth component at GOES 5 caused by the eastward displacement of the flux tube and related negative D deflections on the ground. Figure 15 (bottom) illustrates the dipolarization at GOES 5 caused by the earthward displacement of the flux tube and related negative $\mathrm{H}$ deflections. Although the Hall currents amplified the ground signals, such vector changes of the field lines could be understood in terms of the fundamental harmonic in the toroidal component and the third harmonic in the poloidal component. The polarizations at the satellite footprints can be understood by the different harmonics of the two components.

[17] We propose that dusk-to-dawn currents in the equatorial plane are a possible source for the excitation of the third harmonic in the meridian plane. The dusk-to-dawn currents may correspond to the diamagnetic currents excited by the increased plasma pressures associated with the 

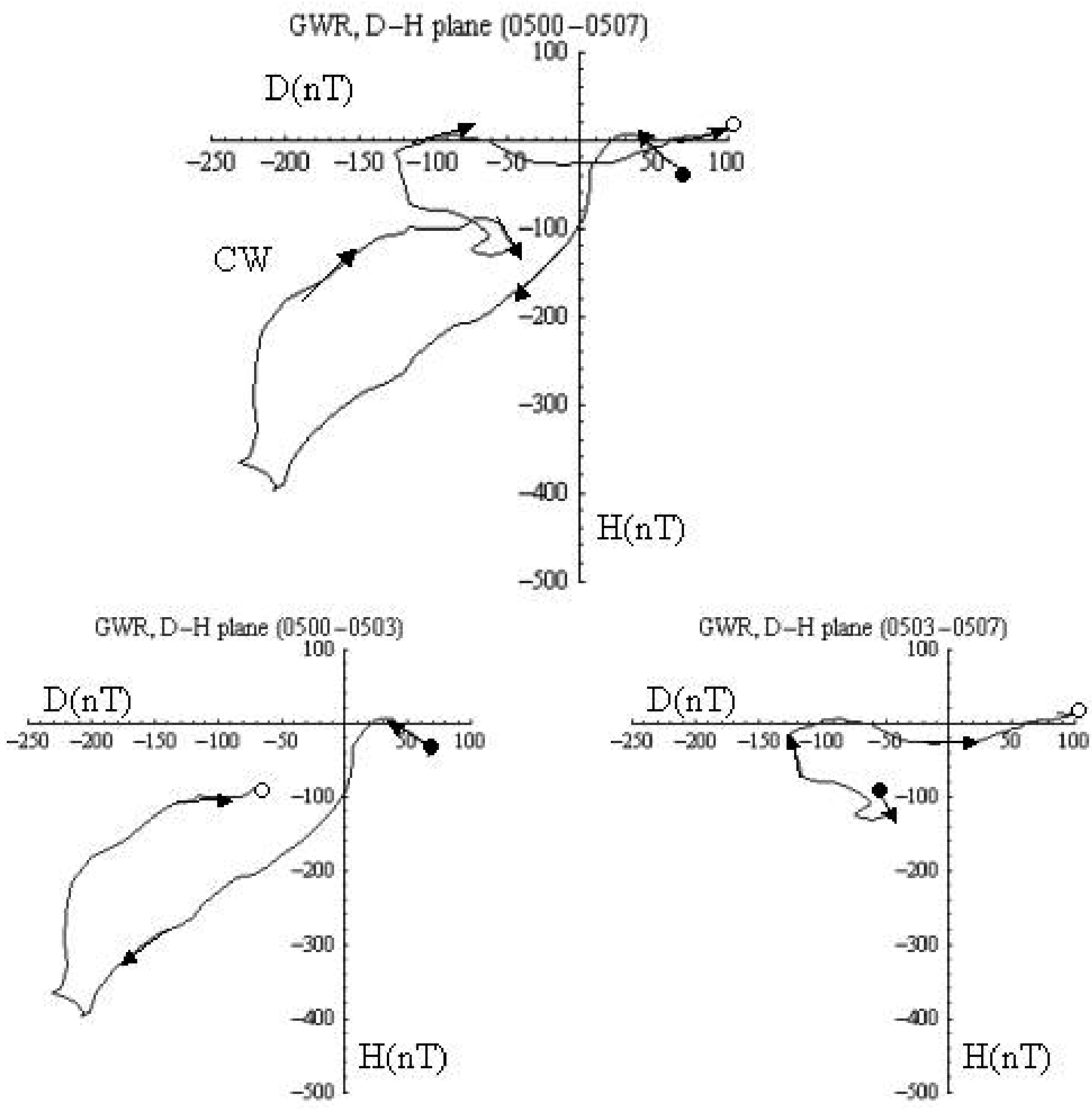

Figure 14. Same as Figure 10 but for the interval 0500-0507 UT for GWR, using unfiltered data. In the bottom panel, the interval 0500-0507 UT is divided into (left) 0500-0503 UT and (right) 0503-0507 UT. The sense of polarization was $\mathrm{CW}$.

passage of the bidirectional flow burst. The diamagnetic currents would be periodically excited by the repetition of the bidirectional flow burst and propagate longitudinally (eastward or westward) at the velocity of the bidirectional flow burst. The diamagnetic currents may be at peak intensity in local time sector where energetic ion injections occurred.

[18] Figure 16 shows how the superposition of the secondary fields produced by the diamagnetic currents modifies the flux densities of the Earth's dipole fields in the meridian planes. Three cases are presented: in Figure 16a the diamagnetic currents are assumed to form a sheet current at an $\mathrm{L}$ between 6 and 7 with a finite thickness of $1 \mathrm{Re}$, in Figure 16b the $\mathrm{L}$ of the diamagnetic currents is between 8 and 9 but the thickness is $1 \mathrm{Re}$, and in Figure 16c the $\mathrm{L}$ of the diamagnetic currents is between 6 and 7 but the thickness is 2 Re. For the current intensities used, the secondary fields at the equatorial plane for the case of Figure $16 \mathrm{a}$ were $0.59 \%$ of the background field magnitudes at $\mathrm{L}=3$. The corresponding amplitude is $6.8 \mathrm{nT}$. Because the secondary field components are mostly parallel to the dipole fields near the equatorial plane and antiparallel off the equatorial plane at the earthward of the diamagnetic currents, distributions of the flux density $(\delta B)$ in the meridian plane are divided into two regions of $\delta B>0$ and $\delta B<0$ as shown by solid bars. The field lines below $\mathrm{L}=3$ remained in the positive $\delta B$ region, while those between $\mathrm{L}=3$ and $\mathrm{L}$ crossing the center of the diamagnetic currents crossed two regions. The field lines beyond the center of the diamagnetic currents remained in the negative $\delta B$ region. We examined the field line deformations of the 

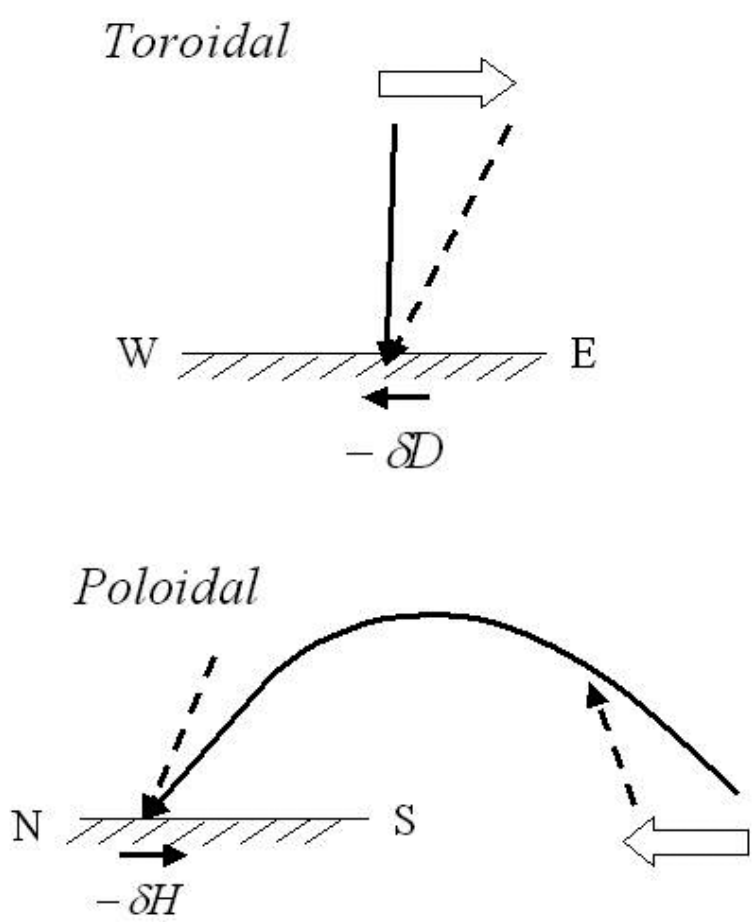

Figure 15. A sketch showing the observed correlations of the changes in field lines at GOES 5 and on the ground for the (top) toroidal and (bottom) poloidal components. The field line changes from solid to dotted arrows in Figure 15 (top and bottom) are associated with the eastward and earthward displacement of the flux tube at GOES 5, respectively (shown by open arrows). On the ground, the changes are westward $(-\delta D)$ and southward $(-\delta H)$ in the $\mathrm{D}$ and $\mathrm{H}$ components, respectively.

three field lines crossing $(X=3 \mathrm{Re}, \mathrm{Z}=0 \mathrm{Re}),(\mathrm{X}=5 \mathrm{Re}, \mathrm{Z}=0$ $\mathrm{Re})$, and $(X=5 \mathrm{Re}, Z=3 \mathrm{Re})$ for different conditions of the diamagnetic currents corresponding to Figures 16a, 16b, and 16c. Results are presented in Figure 17. The intensities of the diamagnetic currents were magnified by 5 to emphasize the deformations. Because the secondary fields rotate the dipole field lines clockwise in the meridian plane viewed from the west, the dipole field lines (solid lines) expand radial outward (dotted lines). The outward expansion becomes less significant for the field lines with lower L. When the field lines were displaced earthward at the equatorial plane by the passage of the bidirectional flow burst, field lines with greater $\mathrm{L}$ are the third harmonic and field lines with lower $\mathrm{L}$ are the fundamental harmonic. The third harmonic tends to be accompanied by a decrease of the field magnitudes at the high-latitudes sector. A sketch of the field line motions in the meridian plane associated with the excitation of the fundamental and third harmonics is presented in Figure 18. The Hall currents at the magnetosphere-atmosphere interface and the ground $\mathrm{H}$ component produced by the Hall currents are also depicted. In the auroral zone where the third harmonic was excited, the Hall currents may form a current loop in the ionosphere propagating either east or west. The ground distributions of polarization $(\mathrm{CW} / \mathrm{CCW})$ in the auroral zone were consistent with the ellipticity map in Figure 7 of Samson and Harrold [1983]. Outside the auroral zone, the ground polarizations may not be interpreted simply by the propagating ionospheric current loop because the third harmonic may not be a major harmonic in the meridian plane.

[19] In the source free magnetosphere, the geomagnetic potential can be expressed in terms of the spherical harmonic series [Mead, 1964]. We assume that the compressional input at the outer boundary is a main contribution to the deformation of geomagnetic fields in the inner magnetosphere. For localized external sources, signals in the inner magnetosphere should be smaller in amplitudes than those at the outer boundary. The amplitude ratio of the substorm Pi2 in the nighttime sector at the dip equator $(\mathrm{L}=1)$ and at geosynchronous altitudes $(\mathrm{L}=6.6)$ was 1:5 [Saka et al., 2010]. The substorm Pi2 may be consistent with the assumption of the localized source. Accounting for such radial dependences, a spherical harmonic series with higher-order terms should be chosen. Consequently, deformation of the geomagnetic fields in the inner magnetosphere would show longitudinal and latitudinal structures. One possible explanation of the mixture of the fundamental and third harmonics is a feature associated with the deformation of the geomagnetic fields by the localized external source.

[20] The diamagnetic current effects that compressed the flux tube would be most significant to Pi2s at the dip equator because the radial component triggered by the external stimulus disappears at the dip equator (HUA) [Allan et al., 1996]. In the auroral zone, a mixture of the diamagnetic current effects may exist causing outward expansion at higher latitudes and inward compression at lower latitudes. Together with the field line motions in the toroidal component triggered by the azimuth flows, the combined field line motions in the auroral zone invoke closed Hall currents in the ionosphere. Propagation of the current loop may produce Pi2s in the auroral zone [e.g., Pashin et al., 1982]. Pi2s in the inner magnetosphere associated with the azimuth flows and those associated with the diamagnetic currents were studied by Saka et al. [1996] and Saka et al. [2002, 2004], respectively.

[21] Takahashi et al. [2003] suggested that the phase differences in the field magnitudes between the ground stations at low latitudes and the satellite at the equatorial plane were 0 or 180 when the satellite was in the plasmasphere or outside the plasmapause, respectively. If we apply the phase relations to the events discussed here, it is unlikely that the plasmapause was beyond the geosynchronous altitudes, with $\mathrm{L}=$ 6.6. However, some Pi2s at $\mathrm{L}=6.6$ indicate the onset inside the plasmasphere during the multiple Pi2 onsets [Saka et al., 2004].

[22] We concluded that the poloidal and toroidal components coupled in the magnetosphere propagate to the ground as Cavity mode. The ground Pi2 signals are ionospheric amplification of these incoming Cavity signals. Fieldaligned currents may not be essential to the amplification process. However, auroral precipitations accompanied by the Pi2s enhance the ionospheric conductivities and spatial inhomogeneities in the auroral zone. The field-aligned currents would have been generated from the enhanced inhomogeneities [e.g., Southwood and Hughes, 1985]. Nevertheless, the field-aligned currents associated with the inhomogeneities 
(A)

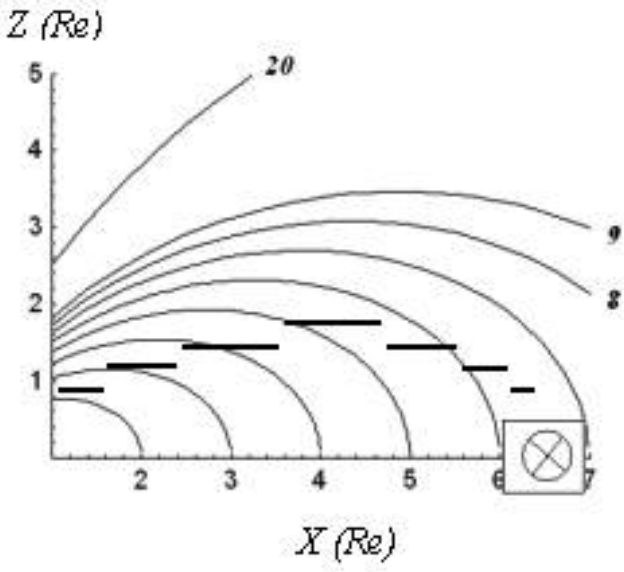

(B)

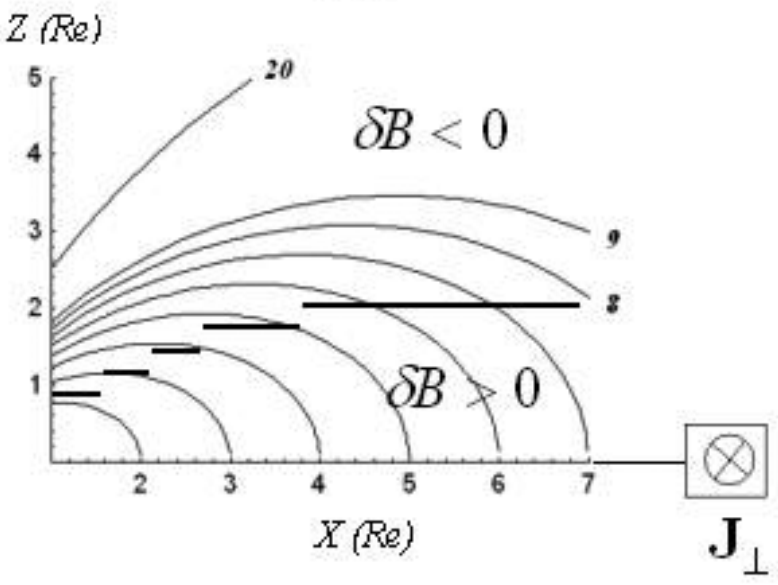

(C)

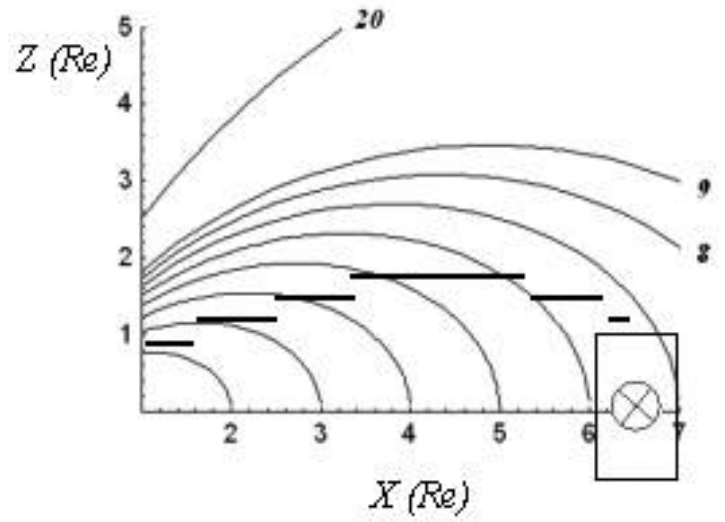

Figure 16. Dipole field lines in the meridian plane corresponding to $\mathrm{L}=2, \mathrm{~L}=3, \mathrm{~L}=4, \mathrm{~L}=5, \mathrm{~L}=6, \mathrm{~L}=7$, $\mathrm{L}=8, \mathrm{~L}=9$, and $\mathrm{L}=20$ illustrated by solid lines. The meridian plane covers $1-7 \mathrm{Re}$ from the center of the Earth in the tailward direction $(\mathrm{X})$ and $0-5 \mathrm{Re}$ from the center of the Earth in the northward direction $(\mathrm{Z})$. Three cases are presented. (a) The dusk-to-dawn currents $\left(\mathbf{J}_{\perp}\right)$ are assumed to be in the equatorial plane between 6 and $7 \mathrm{Re}$ in $\mathrm{X}$. The thickness is $1 \operatorname{Re}(-0.5$ to $+0.5 \operatorname{Re}$ in Z). (b) The thickness of the dusk-todawn currents is $1 \mathrm{Re}$, but the currents are between 8 and $9 \mathrm{Re}$ in $\mathrm{X}$. (c) The dusk-to-dawn currents are between 6 and $7 \operatorname{Re}$ in $X$, but the thickness is $2 \operatorname{Re}(-1.0$ to $+1.0 \operatorname{Re}$ in $Z)$. The flux tube densities increase $(\delta B>0)$ or decrease $(\delta B<0)$ due to the superposition of the secondary fields generated by $\mathbf{J}_{\perp}$. The thick lines divide the meridians into $\delta B>0$ and $\delta B<0$. Field lines beyond the $\mathrm{L}$ of the dusk-to-dawn current remained in the $\delta B<0$ meridian. 
(A)

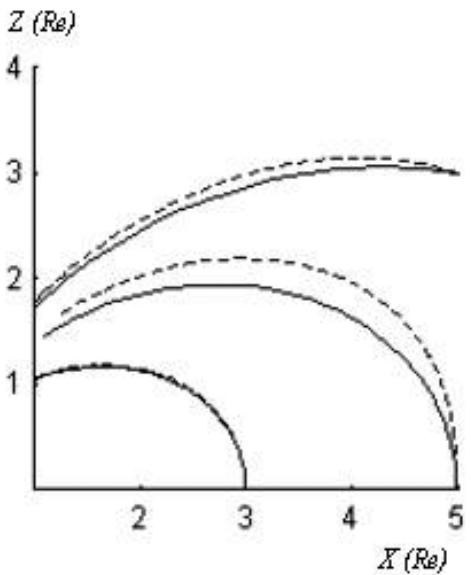

(B)

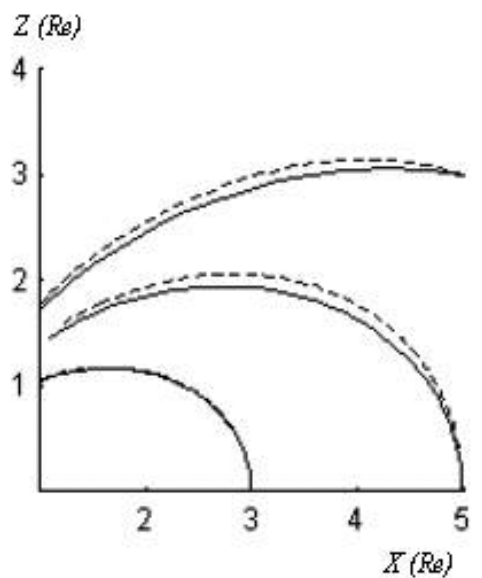

(C)

\section{$Z(R e)$}

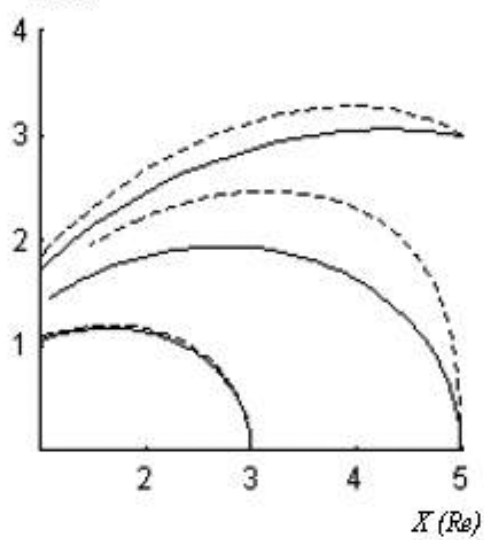

Figure 17. $(\mathrm{a}-\mathrm{c})$ Field line deformations of the dipole field lines caused by the secondary fields of the duskto-dawn currents $\left(\mathbf{J}_{\perp}\right)$ are illustrated in the meridian from 1 to $5 \mathrm{Re}$ in $\mathrm{X}$ and from 0 to $4 \mathrm{Re}$ in $\mathrm{Z}$. Solid lines represent dipole field lines crossing $(X=3 \mathrm{Re}, Z=0 \mathrm{Re}),(X=5 \mathrm{Re}, Z=0 \mathrm{Re})$, and $(X=5 \mathrm{Re}, Z=3 \mathrm{Re})$. Dotted lines illustrate deformation of the dipole field lines. Figures $17 \mathrm{a}-17 \mathrm{c}$ are labeled according to the location and thickness of the $\mathbf{J}_{\perp}$ in Figure 16. In order to emphasize the field line deformations, magnitudes of $\mathbf{J}_{\perp}$ is 5 times larger than that used in Figure 16.

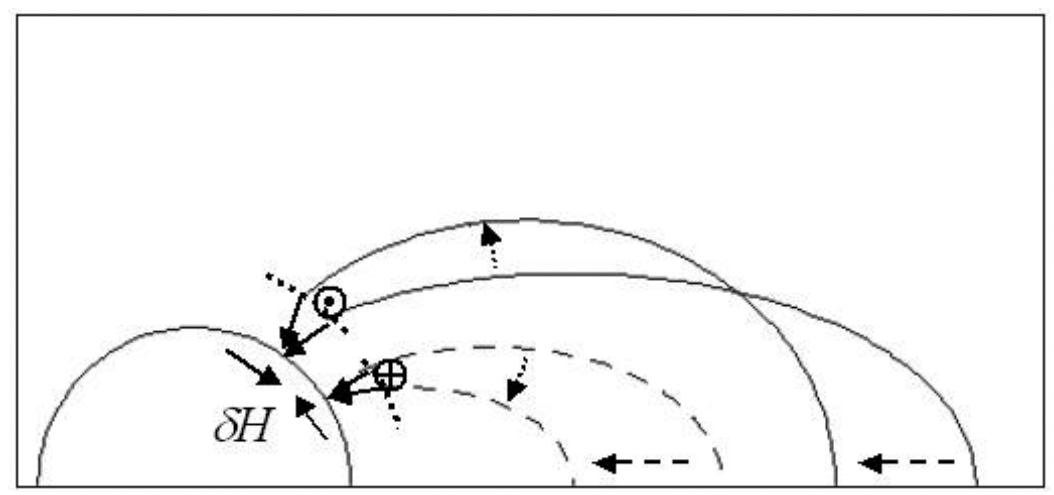

Figure 18. Sketch of field line motions in the midnight meridian of the Northern Hemisphere viewed from the west. Excitation of the fundamental harmonic and the third harmonic are illustrated by dotted and solid curves, respectively, with the field line motions illustrated by dotted arrows. The ionosphere is marked by dotted lines. The ionospheric currents are westward (out of the page) at higher latitudes and eastward (into the page) at lower latitudes associated with the excitation of the third and fundamental harmonics, respectively. The westward and eastward currents produced negative and positive $\delta H$ on the ground, respectively, as shown by the arrows. 
may not significantly modify the ground signals, because the present model adequately explains the ellipticity map of Samson and Harrold [1983].

\section{Summary}

[23] We conclude that the field line motions associated with the third harmonic are excited in the meridian plane, while the fundamental harmonic is excited in the transverse plane in the auroral zone. We propose that the diamagnetic currents in the equatorial plane are related to the generation of the third harmonic. We argue that the diamagnetic currents and the compressional input at the outer boundary are the source of $\mathrm{Pi} 2$ pulsations during the substorm injections. On the ground, the ionospheric Hall currents that cancel the field line discontinuities at the magnetosphere-atmosphere interface amplified the incoming $\mathrm{Pi} 2$ signals.

[24] Acknowledgments. The GOES 5/6 magnetometer data were provided by NOAA, Boulder, Colorado. We thank T. Nagai for the coordinate transform software for the satellite magnetometer data. The IGRF and T89 model calculations were performed through WDC for Aurora in NIPR. We also thank the Institute Geofisico der Peru, Peru, for magnetometer operations at Huancayo.

[25] Robert Lysak thanks the reviewers for their assistance in evaluating this paper.

\section{References}

Allan, W., S. P. White, and E. M. Poulter (1985), Magnetospheric coupling of hydromagnetic waves-Initial results, Geophys. Res. Lett., 12, 287-290, doi:10.1029/GL012i005p00287.

Allan, W., et al. (1996), Are low-latitude Pi2 pulsations cavity/waveguide modes?, Geophys. Res. Lett., 23, 765-768, doi:10.1029/96GL00661.

Baumjohann, W., and K. Glassmeier (1984), The transient response mechanism and Pi2 pulsations at substorm onset-Review and outlook, Planet. Space Sci., 32, 1361-1370, doi:10.1016/0032-0633(84)90079-5.

Keiling, A., and K. Takahashi (2011), Review of Pi2 models, Space Sci. Rev., 161, 63-148, doi:10.1007/s11214-011-9818-4.

Kim, K.-H., et al. (2005), Pi2 pulsations observed from the Polar satellite outside the plasmapause, Geophys. Res. Lett., 32, L18102, doi:10.1029/ 2005GL023872.

Lee, D.-H. (1998), On the generation mechanism of Pi2 pulsations in the magnetosphere, Geophys. Res. Lett., 25, 583-586, doi:10.1029/ 98GL50239.

Lee, D.-H., and R. Lysak (1991), Impulsive excitation of ULF waves in the three-dimensional dipole model: The initial results, J. Geophys. Res., 96 , 3479-3486, doi:10.1029/90JA02349.

Lee, D.-H., and R. Lysak (1999), MHD waves in a three-dimensional dipolar magnetic field: A search for Pi2 pulsations, J. Geophys. Res., 104, 28,691-28,699, doi:10.1029/1999JA900377.

Lester, M., W. J. Hughes, and H. J. Singer (1983), Polarization patterns of Pi2 magnetic pulsations and the substorm current wedge, J. Geophys Res., 88, 7958-7966, doi:10.1029/JA088iA10p07958.

Lester, M., W. J. Hughes, and H. J. Singer (1984), Longitudinal structure in Pi2 pulsations and the substorm current wedge, J. Geophys. Res., 89, 5489-5494, doi:10.1029/JA089iA07p05489.

Li, Y., B. J. Fraser, F. W. Menk, D. J. Webster, and K. Yumoto (1998), Properties and sources of low and very low latitude Pi2 pulsations, J. Geophys. Res., 103, 2343-2358, doi:10.1029/97JA02921.

Mead, G. (1964), Deformation of the geomagnetic field by the solar wind, J. Geophys. Res., 69, 1181-1195, doi:10.1029/JZ069i007p01181.

Oguti, T., T. Kitamura, and T. Watanabe (1988), Global Aurora Dynamics Campaign, 1985-1986, J. Geomagn. Geoelectr., 40, 485-504, doi:10.5636/ jgg.40.485.
Pashin, A. B., et al. (1982), Pi2 magnetic pulsations, auroral break-ups, and the substorm current wedge: A case study, J. Geophys., 51, 223-233.

Saito, T. (1961), Oscillation of geomagnetic field with the progress of pt-type pulsation, science report, pp. 53-61, Tohoku Univ., Sendai, Japan.

Saka, O., H. Akaki, O. Watanabe, and D. N. Baker (1996), Ground-satellite correlation of low-latitude Pi2 pulsations: A quasi-periodic field line oscillation in the magnetosphere, J. Geophys. Res., 101, 15,433-15,440, doi:10.1029/96JA00361.

Saka, O., H. Akaki, and D. N. Baker (2002), A satellite magnetometer observation of dusk-to-dawn current in the midnight magnetosphere at low-latitude Pi2 onset, Earth Planets Space, 54, e1-e4.

Saka, O., H. Akaki, and D. N. Baker (2004), A Pi2-associated dusk-to-dawn currents in the midnight sector as observed at $\mathrm{L}=6.6$ during multiple $\mathrm{Pi} 2$ onsets, Earth Planets Space, 56, 663-668.

Saka, O., D. Koga, and K. Hayashi (2007), A plasma bulk motion in the midnight magnetosphere during auroral breakup inferred from all-sky image and magnetic field observations at geosynchronous altitudes, J. Atmos. Sol. Terr. Phys., 69, 1063-1074, doi:10.1016/j.jastp.2007. 04.003.

Saka, O., K. Hayashi, and M. Thomsen (2010), First 10 min intervals of $\mathrm{Pi} 2$ onset at geosynchronous altitudes during the expansion of energetic ion regions in the nighttime sector, J. Atmos. Sol. Terr. Phys., 72, 1100-1109, doi:10.1016/j.jastp.2010.07.006.

Saka, O., K. Hayashi, and D. Koga (2012), Periodic aurora surge propagating eastward/westward at poleward boundary of aurora zone during the first $10 \mathrm{~min}$ intervals of Pi2 onset, J. Atmos. Sol. Terr. Phys., 80, 285-295, doi:10.1016/j.jastp.2012.02.010.

Samson, J. (1982), Pi2 pulsations: High latitude results, Planet. Space Sci., 30, 1239-1247, doi:10.1016/0032-0633(82)90097-6.

Samson, J., and B. Harrold (1983), Maps of the polarizations of high latitude Pi2's, J. Geophys. Res., 88, 5736-5744, doi:10.1029/JA088iA07p05736.

Samson, J., and B. Harrold (1985), Characteristic time constants and velocities of high-latitude Pi2's, J. Geophys. Res., 90, 12,173-12,181, doi:10.1029/JA090iA12p12173.

Shinohara, M., K. Yumoto, A. Yoshikawa, O. Saka, S. I. Solovyev, E. F. Vershinin, N. B. Trivedi, J. M. Da Costa, and The $210^{\circ}$ MM Magnetic Observation Group (1997), Wave characteristics of daytime and nighttime Pi2 pulsations at the equatorial and low latitudes, Geophys. Res. Lett., 24, 2279-2282, doi:10.1029/97GL02146.

Southwood, D. J., and W. J. Hughes (1985), Concerning the structure of Pi2 pulsations, J. Geophys. Res., 90, 386-392, doi:10.1029/JA090iA01p00386.

Takahashi, K., S.-I. Ohtani, and K. Yumoto (1992), AMPTE CCE observations of Pi2 pulsations in the inner magnetosphere, Geophys. Res. Lett., 19, 1447-1450, doi:10.1029/92GL01283.

Takahashi, K., D.-H. Lee, M. Nosé, R. R. Anderson, and W. J. Hughes (2003), CRRES electric field study of the radial mode structure of Pi2 pulsations, J. Geophys. Res., 108(A5), 1210, doi:10.1029/2002JA009761.

Teramoto, M., M. Nosé, and P. R. Sutcliffe (2008), Statistical analysis of $\mathrm{Pi} 2$ pulsations inside and outside the plasmasphere observed by the polar orbiting DE-1 satellite, J. Geophys. Res., 113, A07203, doi:10.1029/ 2007JA012740.

Tsyganenko, N. A. (1989), A magnetospheric magnetic field model with a warped tail current sheet, Planet. Space Sci., 37, 5-20, doi:10.1016/00320633(89)90066-4.

Uozumi, T., K. Yumoto, H. Kawano, A. Yoshikawa, J. V. Olson, S. I. Solovyev, and E. F. Vershinin (2000), Characteristics of energy transfer of Pi2 magnetic pulsations: Latitudinal dependence, Geophys. Res. Lett., 27, 1619-1622, doi:10.1029/1999GL010767.

Uozumi, T., et al. (2004), Propagation characteristics of Pi2 magnetic pulsations observed at ground high latitudes, J. Geophys. Res., 109, A08203, doi:10.1029/2003JA009898.

Wygant, J. R., et al. (2000), Polar spacecraft based comparisons of intense electric fields and pointing flux near and within the plasma sheet-tail lobe boundary to UVI imagers: An energy source for the aurora, J. Geophys. Res., 105, 18,675-18,692, doi:10.1029/1999JA900500. 